\title{
Oligonucleotide-based targeted gene editing in C. elegans via the CRISPR/Cas9 system
}

\author{
Cell Research (2014) 24:247-250. doi:10.1038/cr.2014.9; published online 14 January 2014
}

\section{Dear Editor,}

Technologies to achieve specific and precise genome editing, such as knock-in and knock-out, are critical for deciphering the functions of a gene and for understanding fundamental biological processes. Compared with the zinc finger nucleases (ZFN) and transcription activator-like effector nucleases (TALEN), which have been used for genome editing [1], the Clustered Regularly Interspaced Short Palindromic Repeats (CRISPR)/ CRISPR-associated (Cas) system has emerged as a new powerful tool for genome modifications. It has recently been adopted for genome editing in human cell lines [24], mouse [5], zebrafish [6], C. elegans [7-12], and plants [13].

In the widely used CRISPR/Cas9 system [2-4], the Cas9 endonuclease is ushered to the specific site of interest by the single guide RNA (sgRNA), an engineered fusion molecule of the targeting CRISPR RNA (crRNA) with the trans-activating crRNA, to generate doublestranded DNA breaks (DSDBs) in the target site. The DSDBs can be repaired either through non-homologous end joining (NHEJ), which leads to generation of random deletions, insertions, or both (InDels) [2-5, 7-9, 11, 13], or through homologous recombination (HR), which could generate specific and precise nucleotide or sequence replacements $[3,5,9,10,12]$ when a plasmid or a single-stranded oligonucleotide (oligo) template is also present. The use of oligonucleotides as donor templates, which can be rapidly synthesized through commercial sources, to achieve Cas9-mediated knock-ins has not yet been reported in C. elegans.

We demonstrate here that oligos can be used as templates in the CRISPR/Cas9 system to generate precise single-nucleotide changes in the $C$. elegans genome (Figure 1A). We used the Peft-3::cas $9:: S V 40$ NLS::tbb-2 3' UTR vector and sgRNA driven by the $C$. elegans U6 promoter to ensure stable and efficient expression of the cas 9 gene and sgRNA in the C. elegans germline [7]. sgRNAs were designed to target sequences of interest in the form of $\mathrm{G} / \mathrm{A}(\mathrm{N})_{19}$, which precede the NGG proto- spacer-adjacent motif (PAM) in the target sites [2-4]. The donor oligonucleotide contains the desired nucleotide change(s) flanked by approximately 50 nucleotides on both sides that match the target sequence (Supplementary information, Table S1).

We first made sgRNAs to target sequences in the unc119 and sup-17 genes and corresponding donor oligos to correct point mutations in the unc-119(ed3) and sup17(n1258) mutants, respectively (Figure $1 \mathrm{~B}$ and 1C). unc-119(ed3) is a recessive nonsense mutation that causes an uncoordinated (Unc) defect. sup-17(n1258) is a recessive missense mutation (V473D) that results in a temperature-sensitive lethality phenotype. These two mutants are used to facilitate identification of correctly edited animals that become phenotypically wild type.

We injected unc-119(ed3) animals with Peft$3::$ cas $9:: S V 40$ NLS::tbb-2 3' UTR, PU6::sgRNA, the donor oligo, and Pmyo-3::mCherry as a transgenic marker (Supplementary information, Data S1). No wild-type or non-Unc animal was seen in 80 mCherry-positive, firstgeneration transgenic progeny isolated (defined as F1), which would have occurred if one of the unc-119(ed3) chromosomes had been correctly edited. However, we did identify a non-Unc heterozygous F1 animal that did not express the mCherry transgenic marker and thus was a non-transgenic F1 progeny (Figure 1D). The T-to$\mathrm{C}$ nucleotide change that corrects the ed 3 mutation was confirmed by DNA sequencing (Figure 1B and Supplementary information, Figure S1). Because this F1 animal did not carry the cas 9 -containing extrachromosomal transgene, it might have inherited the Cas9 protein and sgRNA synthesized in the germline of its mother. The single-stranded oligo that was injected into the germline of the mother but not integrated into the transgene array was likely also passed to this F1 animal and then served as a template for repairing the DSDB. The unexpected finding that a correctly edited unc-119(ed3) animal was obtained from non-transgenic F1 progeny indicates that the current strategy of screening for correctly edited animals from transgenic progeny in C. elegans, albeit proven to be the most efficient one for isolating InDels [7, 
A

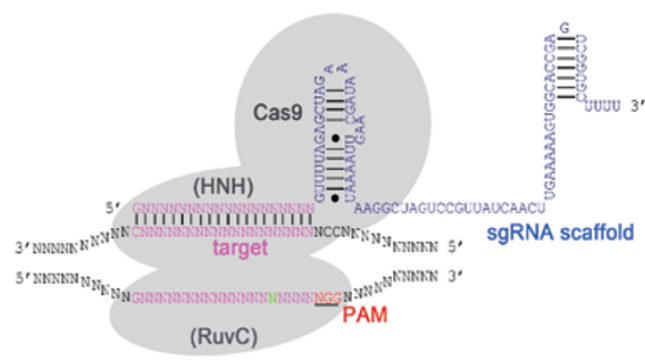

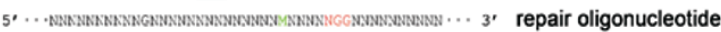

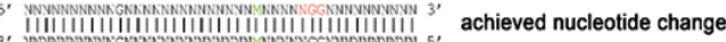

D

\begin{tabular}{lccc}
\hline \multicolumn{1}{c}{ Genotype } & $\begin{array}{c}\text { Injected } \\
\text { animals }\end{array}$ & $\begin{array}{c}\text { Knock-in from } \\
\text { transgenic F1 }\end{array}$ & $\begin{array}{c}\text { Knock-in from } \\
\text { non-transgenic F1 }\end{array}$ \\
\hline unc-119(ed3) & 100 & $0 / 80$ & $1 / \mathrm{ND}$ \\
sup-17(n1258)* & 100 & $0 / 95$ & $1 / 125$ \\
ben-1(sm296) & 30 & $0 / 128$ & $5 / 142$ \\
mec-4(u231) & 15 & $0 / 149$ & $1 / 146$ \\
\hline
\end{tabular}

E

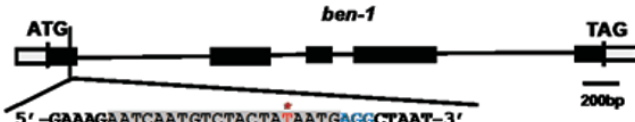

3'-CTTTCTTAGTTACAGATGATATTACTCCEATTA-5'

5' - GAAAGAATCAATGTCTACTAĞ AATGAGGCTAAT-3 T $\rightarrow G$ 5' - GANAGAATCAATGTCTACTAC̆́AATGAGGCTAAT-3' 5' - GAAAGAATCAATGTCTACTAËAACGANGCTAAT-3' T $\rightarrow G$, two 1bp replacements 5' - GAAAGAATCAATGTCTACTATA-TGAGCCTAAT-3 -1bp 5' -GAAACAATCAATGTCTACTATA-TGAGGCTAAT-3' -16p 5' -GAAAGAATCAATGTCTACT-_- TGAGGCTAAT-3' -4bp, +11bp MAGGCTAATGG
B

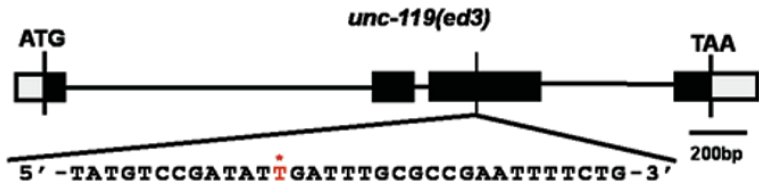

3' -ATACA GGCTATAACTAAACGCGGCTTAAAAGAC -5'

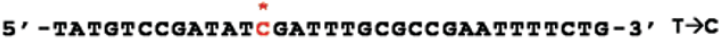

C

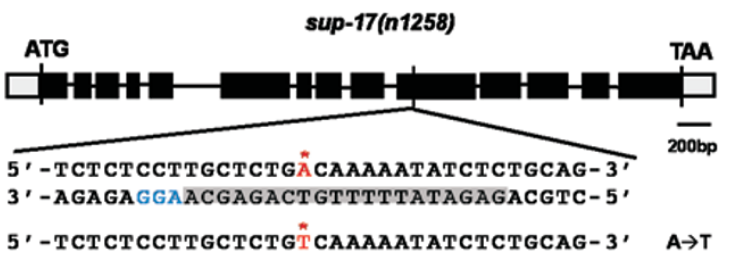

$\mathbf{F}$

\begin{tabular}{cccc}
\hline $\begin{array}{c}\text { F1 } \\
\text { animals }\end{array}$ & BenomylR/F1 & $\begin{array}{c}\text { Knock-ins/ } \\
\text { sequenced }\end{array}$ & $\begin{array}{c}\text { Precise knock- } \\
\text { ins/sequenced }\end{array}$ \\
\hline $\begin{array}{c}\text { Cas9 } \\
\text { transgenic }\end{array}$ & $16 / 45$ & $2 / 5$ & $2 / 5$ \\
$\begin{array}{c}\text { Non- } \\
\text { transgenic }\end{array}$ & $19 / 219$ & $3 / 6$ & $2 / 6$ \\
\hline
\end{tabular}

G

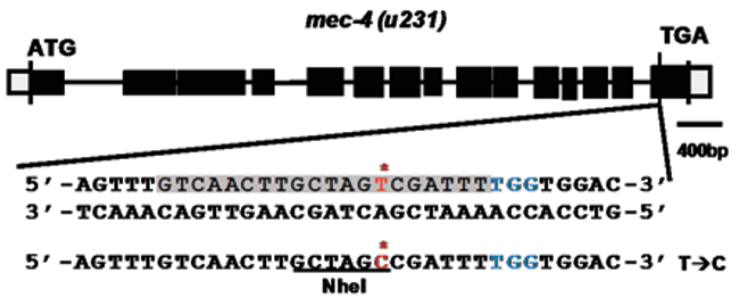

Figure 1 Oligonucleotide-based gene editing in C. elegans. (A) A schematic diagram of the CRISPR/Cas 9 system using an oligonucleotide as the repair template. sgRNA directs Cas 9 to the target site where Cas 9 cleaves the DNA sequence preceding the PAM sequence. The oligonucleotide, containing a single nucleotide change (indicated as $\mathrm{M}$ ), serves as a template to repair the DSDB via HR. (B, C, G) Gene editing at the unc-119(ed3), sup-17(n1258) and mec-4(u231) loci. The sense or anti-sense DNA sequences targeted by sgRNAs are shaded in gray, and the PAM sequences in blue. The nucleotide targeted for substitution is in red and also marked with a red asterisk. The Nhel site generated in $\mathbf{G}$ is underlined. (D) Summary of gene-editing experiments at 4 different genetic loci. *: a phosphorothioate-modified oligo was used as a repair template. ND: not determined. (E) Gene editing at the ben-1 locus. The sense-strand sequence targeted by sgRNA is shaded in gray. The nucleotide targeted for substitution is labeled in red and marked with a red asterisk. DNA sequences in the target region from homozygous benomyl-resistant progeny derived from non-transgenic F1 animals are aligned with the wild-type DNA sequence, with unanticipated nucleotide substitutions (green), deletions, and insertion indicated. (F) Summary of ben-1 gene editing. The numbers of benomyl-resistant (benomyl $\left.{ }^{R}\right) \mathrm{F} 1$ animals versus the number of Cas 9 transgenic or non-transgenic F1 animals are shown. The number of F1 animals with the desired Amber mutation (knock-ins) and the number of F1 animals with the correct Amber mutation and no other mutations in the ben-1 gene (precise knock-ins) versus the number of benomyl ${ }^{R} \mathrm{~F} 1$ animals sequenced are also shown.

9, 10], may not apply to oligo-based gene editing.

Consistently, our attempt to obtain a sup-17(n1258)to-wild-type revertant from Cas 9 transgenic progeny through oligo-based editing did not succeed (Supplementary information, Data S1). As phosphorothioatemodified oligonucleotides have been used in Xenopus embryos to achieve better gene silencing [14], probably due to improved oligo stability in vivo, we tested whether phosphorothioate-modified oligonucleotides (p-oligos) can be used in C. elegans to increase the efficiency of gene editing. We failed to recover any wild-type revertant from mCherry-positive, Cas9 transgenic F1 animals using the sup-17 p-oligo as a repair template (Figure 1D and Supplementary information, Table S1). However, we did recover many wild-type F2 progeny from one nontransgenic $\mathrm{F} 1$ animal at $25{ }^{\circ} \mathrm{C}(1 / 125$; Figure 1D), the 
non-permissive temperature for the sup-17(n1258) mutant. Sequencing results confirmed that sup-17(n1258) was indeed corrected back to the wild-type sequence (Figure 1C and Supplementary information, Figure S2), providing additional evidence that non-transgenic F1 progeny can produce correctly edited animals in oligobased gene editing experiments.

Having successfully converted ed 3 and $n 1258$ mutations into wild-type sequences, we applied this oligobased gene editing method to introduce mutations into wild-type animals, an essential step in analysis of gene functions. We designed an sgRNA to target the ben-1 gene (Figure 1E) and a donor oligo to introduce a nonsense mutation, the Amber stop, at Tyrosine 51 in the first exon of ben-1 (Figure 1E). ben- 1 encodes a $\beta$-tubulin that is sensitive to the treatment of benomyl (an anti-microtubule drug) [15], which leads to slow growth and paralysis of animals at $25{ }^{\circ} \mathrm{C}$. As ben-1 loss-of-function mutations are dominant suppressors of the benomyl-induced paralysis or Unc defect [15], we could easily identify mutated $\mathrm{F} 1$ heterozygous or homozygous animals placed on $14 \mathrm{mM}$ benomyl plates. From 5 wild-type C. elegans animals (N2 strain) injected with the oligo-containing mixture (Supplementary information, Data S1), we identified 16 non-Unc animals from 45 Cas 9 transgenic F1 animals (mCherry positive) and 19 non-Unc animals from 219 non-transgenic F1 animals (Figure 1F). Homozygous non-Unc $\mathrm{F} 2$ progeny were isolated from nonUnc F1 animals and the entire ben-1 locus of some F2 progeny was sequenced to confirm the presence of the Amber mutation and to identify other potential mutations. Among 5 randomly selected transgenic non-Unc $\mathrm{F} 1$ animals, we found 2 animals carrying the right Amber mutation and no other mutation in the ben-1 gene (Figure 1F and Supplementary information, Figure S3). The other 3 transgenic non-Unc F1 animals did not contain the desired Amber mutation, and instead, had 2-bp, 7-bp and 354-bp deletions at or near the targeted site, respectively (Supplementary information, Figure S3). We also sequenced the homozygous progeny of 6 non-transgenic F1 animals and identified $2 \mathrm{~F} 1$ animals carrying the right Amber mutation and no other mutation in the ben-1 gene (Figure 1E and Supplementary information, Figure S4), one of which actually had both ben-1 copies edited correctly as all of its F2 progeny are non-Unc animals. Among the other 4 examined non-transgenic F1 animals, one is homozygous for the Amber mutation but with 2 additional 1-bp substitutions (Figure 1E and Supplementary information, Figure S4), and the other three do not harbor the Amber mutation but contain InDels of various kinds in the targeted region (Figure 1E and Supplementary information, Figure S4). Together, these results dem- onstrate that precise oligo-based gene editing can occur in both Cas9 transgenic and non-transgenic animals.

In the above ben-1 gene editing experiments, at least 4 precisely edited $\mathrm{F} 1$ animals were obtained from five injected N2 animals (Figure 1F). By contrast, only one correctly edited F1 animal was obtained from 100 injected unc-119(ed3) or sup-17(n1258) animals (Figure 1D). This is probably due to the fact that unc-119(ed3) and sup-17(n1258) animals are not as healthy as N2 animals and have smaller brood sizes and abnormal gonad morphology that causes difficulty for microinjection. Therefore, more animals need to be injected to produce a sufficient amount of F1 progeny.

We also tried to revert the newly generated ben-1 Amber mutation, sm296, back to the wild-type sequence (Supplementary information, Figure S5), which would cause paralysis of the correctly edited homozygous animals upon benomyl treatment. From 30 injected ben1(sm296) animals, we did not observe any Unc animal in 128 Cas 9 transgenic F1 animals or their F2 progeny, but identified 5 heterozygous $\mathrm{F} 1$ animals producing paralyzed F2 progeny from 142 non-transgenic F1 animals (Figure 1D). Sequencing analyses of homozygous Unc progeny from these 5 heterozygous F1 animals revealed correct editing of the Amber mutation back to the wildtype sequence (Supplementary information, Figure S5). These results further indicate that non-transgenic F1 animals are more likely to have precise nucleotide changes in the genome through oligo-based gene editing than Cas9 transgenic F1 animals.

The above gene-editing experiments rely on screening for modified animals with easily identifiable phenotypes, such as Unc/non-Unc and embryonic lethality/viable adults. To expand the utility of this oligo-based geneediting method, we used the single nucleotide polymorphism (SNP) method to screen for modified animals that have subtle or no detectable phenotypes. We attempted to revert the mec-4(u231) allele (A713V), which causes necrotic death of six mechanosensory neurons, to the wild-type allele. An sgRNA and a donor oligo were designed to target the $u 231$ site in the mec-4 gene to revert the mutation (GTC) back to the wild-type sequence (GCC) (Figure 1G), which would generate an NheI restriction digestion site (gctaGCC) that is absent in the u231 sequence (gctaGTC; Figure $1 \mathrm{G}$ and Supplementary information, Table S1). A 1025-bp genomic fragment spanning the sgRNA-targeted site in the mec-4 gene was PCR amplified from N2 and mec-4(u231) animals and digested with NheI. The PCR products derived from mec4(u231) animals could not be cleaved by NheI, whereas the PCR products from N2 animals yielded 382-bp and 643-bp fragments after the NheI digestion (Supplementary 
information, Figure S6A). From 15 injected mec-4(u231) animals, we screened 149 Cas9 transgenic F1 animals and 146 non-transgenic F1 animals by PCR analysis and NheI digestion. None of the Cas9 transgenic F1 animals produced PCR products that could be cleaved by NheI, whereas one heterozygous non-transgenic F1 animal produced PCR products that were partially digested by NheI to generate 2 fragments of correct sizes (Figure 1D, Supplementary information, Figure S6A and Data S1). Sequencing analysis of homozygous progeny from this heterozygous F1 animal confirmed correct editing at the mec-4(u231) locus (Figure 1G and Supplementary information, Figure S6B-S6D). Therefore, this oligo-based gene editing method can be broadly used to generate precise nucleotide changes at sites where an SNP can be identified by restriction digestion, and potentially, can be used at any sgRNA-targetable site in the genome, when combined with a mismatch-specific endonuclease such as the CEL-1 endonuclease [9].

Interestingly, our results indicate that precise genome editing is more likely to occur in non-transgenic F1 animals than in Cas9 transgenic ones in this oligo-based approach (Figure 1D). We suspect that the continuous expression of both Cas9 and sgRNA in the germline of transgenic F1 animals may lead to multiple cleavage events in the sgRNA-targeted region, which would facilitate generation of InDels but be detrimental to precise gene editing via HR. Indeed, when we tried to generate mec-4(u231) mutations in N2 animals, which would destroy the NheI site in the mec-4 gene, all 4 F1 animals heterozygously missing the NheI site identified through restriction analysis (from 256 Cas9 transgenic F1 animals) contained deletions in the targeted region (Supplementary information, Figure S7), three of which directly removed the nucleotide targeted for substitution.

To our knowledge, this is the first study employing oligonucleotides as templates to successfully generate precise nucleotide changes in the $C$. elegans genome via the CRISPR/Cas9 system. Moreover, we report the unexpected finding that precise genome editing occurs more frequently in Cas9 non-transgenic F1 progeny. Compared with other gene editing methods that require construction of double-stranded DNA templates, this oligo-based method allows rapid and seamless editing of the genome at precise locations and can become a powerful tool for probing the functions of genes or motifs, for altering critical residues in proteins to create desirable gain-offunction or loss-of-function mutations, or for generating mutations in highly conserved proteins in C. elegans to facilitate the study of corresponding human diseases.

Detailed methods are described in Supplementary information, Data S1.

\section{Acknowledgments}

We thank Yu Peng, Man Zhang, and Qian Liang for discussion. This work was supported by the Tsinghua University-Peking University Center for Life Sciences, the National Basic Research Program of China (973 Program, 2013CB945602) and NIH (R01GM59083).

Pei Zhao ${ }^{1, *}$, Zhe Zhang ${ }^{1, *}$, Hongmei Ke ${ }^{1}$, Yiren Yue ${ }^{1}$, Ding Xue ${ }^{1,2}$

${ }^{I}$ School of Life Sciences, Tsinghua University, Beijing 100084, China; ${ }^{2}$ Department of Molecular, Cellular, and Developmental Biology, University of Colorado, Boulder, Colorado 80309, USA

*These two authors contributed equally to this work.

Correspondence: Ding Xue

E-mail: ding.xue@colorado.edu

\section{References}

1 Mussolino C, Cathomen T. Curr Opin Biotechnol 2012; 23:644-650.

2 Mali P, Yang L, Esvelt KM, et al. Science 2013; 339:823-826.

3 Cong L, Ran FA, Cox D, et al. Science 2013; 339:819-823.

4 Jinek M, East A, Cheng A, et al. eLife 2013; 2:e0471.

5 Wang H, Yang H, Shivalila CS, et al. Cell 2013; 153:910-918.

6 Hwang WY, Fu Y, Reyon D, et al. Nat Biotechnol 2013; 31:227-229.

7 Friedland AE, Tzur YB, Esvelt KM, et al. Nat Methods 2013; 10:741743.

8 Chiu H, Schwartz HT, Antoshechkin I, et al. Genetics 2013; 195:11671171.

9 Lo TW, Pickle CS, Lin S, et al. Genetics 2013; 195:331-348.

10 Dickinson DJ, Ward JD, Reiner DJ, et al. Nat Methods 2013; 10:10281034.

11 Waaijers S, Portegijs V, Kerver J, et al. Genetics 2013; 195:1187-1191.

12 Chen C, Fenk LA, de Bono M. Nucleic Acids Res 2013; 41:e193.

13 Feng Z, Zhang B, Ding W, et al. Cell Res 2013; 23:1229-1232.

14 Lennox KA, Sabel JL, Johnson MJ, et al. Oligonucleotides 2006; 16:26-42.

15 Driscoll M, Dean E, Reilly E, et al. J Cell Biol 1989; 109:2993-3003.

(Supplementary information is linked to the online version of the paper on the Cell Research website.) 